\title{
Preoperative Predictors of Extended Hospital Length of Stay Following Total Knee Arthroplasty
}

\author{
Mohamad J. Halawi, MD ${ }^{\text {a }}$, Tyler J. Vovos ${ }^{\mathrm{b}}$, Cindy L. Green, $\mathrm{PhD}^{\mathrm{c}}$, Samuel S. Wellman, MD ${ }^{\mathrm{a}}$, \\ David E. Attarian, MD ${ }^{\mathrm{a}}$, Michael P. Bolognesi, MD ${ }^{\mathrm{a}}$ \\ a Department of Orthopaedic Surgery, Duke University Medical Center, Durham, North Carolina \\ b Duke University School of Medicine, Durham, North Carolina \\ c Department of Biostatistics and Bioinformatics, Duke University Medical Center, Durham, North Carolina
}

\section{A R T I C L E I N F O}

\section{Article history:}

Received 3 September 2014

Accepted 16 October 2014

\section{Keywords:}

knee

arthroplasty

length of stay

preoperative predictors

assistance at home

comorbidities

\begin{abstract}
A B S T R A C T
The purpose of this study was to identify the preoperative predictors of hospital length of stay after primary total knee arthroplasty in a patient population reflecting current trends toward shorter hospitalization and using readily obtainable factors that do not require scoring systems. A single-center, multi-surgeon retrospective chart review of two hundred and sixty consecutive patients who underwent primary total knee arthroplasty was performed. The mean length of stay was 3.0 days. Among the different variables studied, increasing comorbidities, lack of adequate assistance at home, and bilateral surgery were the only multivariable significant predictors of longer length of stay. The study was adequately powered for statistical analyses and the concordance index of the multivariable logistic regression model was 0.815 .
\end{abstract}

(c) 2014 Elsevier Inc. All rights reserved.
Total knee arthroplasty (TKA) has revolutionized the treatment for end-stage knee arthritis with mounting evidence demonstrating its reliability and cost-effectiveness [1]. By 2030, the number of primary TKA in the United States is estimated to increase by nearly seven-fold to 3.48 million procedures [2]. In contrast, the number of hospital beds nationwide has steadily decreased since 1975 [3]. This demand-supply mismatch in total joint hospital beds combined with nursing shortages [4] implores the need for early and accurate prediction of hospital length of stay (LOS) to ensure efficient and cost-effective provision of health services.

In recent years, the LOS following TKA in the U.S. has decreased substantially, in part due to improvements in surgical techniques including minimally invasive approaches [5] and adoption of postoperative clinical pathways emphasizing multimodal analgesia and rapid mobilization [6-8]. Medicare's prospective payment system is another incentive for shorter LOS. Medicare provides fixed payments to hospitals based on diagnosis related groups [9]. The longer patients stay, the more money hospitals lose. Among Medicare beneficiaries, the mean LOS has decreased from 7.9 days in 1991 to 3.5 days in 2010 [10].

While studies examining the risk factors for prolonged hospitalization have previously been published, several factors limit their application to current TKA practice in the United States, particularly in surgical centers emphasizing shorter hospitalization. Among those limitations

The Conflict of Interest statement associated with this article can be found at http:// dx.doi.org/10.1016/j.arth.2014.10.025.

Reprint requests: Mohamad J. Halawi, MD, Department of Orthopaedic Surgery, Duke University Medical Center Box 3000, Durham, NC 27710. are significantly long LOS [11], lack of physical therapy on weekends $[11,12]$, postoperative rehabilitation criteria geared toward home-only discharge [12,13], focus on select medical comorbidities [14], inclusion of procedures other than TKA in the analysis [14], and broad exclusion criteria [15]. Furthermore, factors such as preoperative pain level and patient expectation were often not investigated.

Predicting LOS following TKA is important for resource allocation, discharge planning, and operational efficiency. Early prediction of LOS may also help manage patient expectations and has implications for healthcare costs. Building on previous findings, the aim of this study was to identify preoperative predictors of LOS after primary TKA in a patient population reflecting current trends toward shorter hospitalization and using readily obtainable factors that do not require scoring systems. We also sought to investigate patient factors not previously studied, particularly the contribution of preoperative pain level and patient expectation to LOS.

\section{Material and Methods}

IRB approval was obtained. The primary measure of the study was LOS, defined as the number of nights from admission to discharge. Prolonged hospitalization was defined as LOS $>3$. This control value was selected for three reasons: 1 ) it represented the standard target for discharge in our practice, 2 ) it was the mean LOS in our patient sample, and 3 ) it reflected current national trends [10]. All patients admitted to our institution between January 1, 2012 and December 31, 2012 with CPT code 27447 (total knee arthroplasty) as the primary procedure 
were included in the study. Two hundred and sixty patients were identified and their medical records were retrospectively reviewed. Three surgeons performed the operations using the standard medial parapatellar approach and a variety of cemented implants. The patella was routinely resurfaced. While Medicare beneficiaries made up over half the patient population, patients of all payor types were included.

All patients were admitted from home on the day of surgery. Spinal anesthesia and continuous femoral nerve blocks (FNB) were used unless contraindicated. Following TKA, patients had a standardized clinical pathway with regards to pain control, mobilization, and anticoagulation. FNB and intravenous patient-controlled analgesia were provided immediately following surgery and discontinued on the first postoperative day. Given the risk of fall associated with FNB-induced quadriceps weakness, knee immobilizers were applied until discontinuation of FNB to aid with ambulation during initial physical therapy (PT) visits. PT was initiated on the day of surgery and continued daily until discharge. The primary goals of the first PT/OT session were to assess patients' needs, review acute rehabilitation goals, assist with mobility as tolerated, and initiate preliminary discharge planning. As part of the preliminary discharge planning, the therapists inquired about patients' expectation of discharge destination, which was grouped into either home or extended care facility (acute rehabilitation and skilled nursing facilities). During the period the knee arthroplasties in this study were performed, patients were provided with informational packets in clinic describing the procedure, benefits, complications, and rehabilitation. They were also given the option of attending a total joint camp, but participation was low and did not exceed $20 \%$. However, patients were informed by their surgeons that the target day of discharge to home is the second postoperative day and to an extended care facility (ECF) the third postoperative day. They were also informed that the decision for discharge to home vs. ECF primarily depended on their progress with PT/OT.

Unless contraindicated, enoxaparin was used for DVT prophylaxis. Patients were cleared for discharge when they were medically stable, had adequate pain control, were able to void and tolerate oral diet, had no surgical concerns, and were functionally suitable for their discharge destination as determined by PT/OT. At our institution, we do not have policies that mandate the LOS TKA patients, except for the requirement of a 3-day stay for Medicare beneficiaries needing discharge to ECF. In addition, there were no de facto expectations that our Medicare population would be discharged to an ECF.

The preoperative factors analyzed were age, gender, body mass index (BMI), number of comorbidities, history of prior total hip or knee arthroplasty, history of prior admission to an ECF, pain level, use of assistive devices, type of surgery ( unilateral or bilateral), caregiver assistance at home, and patient expectation of discharge destination. A comorbid condition was defined as any condition (medical or mental) that was actively treated at the time of surgery. Because a majority of patients carried a diagnosis of arthritis and were receiving medications for joint pain, arthritis was counted as a comorbid condition only if it involved joints other than the replaced knee(s). We intentionally chose to assess health status based on the number of active conditions in order to eliminate the use of scoring systems, which was one of the goals of this study. Caregiver assistance was classified as either adequate for patient needs (meals, hygiene, etc.) or inadequate. Pain level was assessed at the time of preoperative anesthesia evaluation and collected using the numeric rating scale for pain. Patient expectation of discharge destination was collected at the time of initial PT/OT session as described above.

Results were summarized using the mean and standard deviation for continuous variables and percentages for categorical variables. The relationship between each categorical variable and LOS $>3$ days was assessed using the chi-square test, or in the presence of small cell counts, Fisher's exact test. Analysis of continuous variables was performed using Student's t-test. All statistical tests were two-sided, and a $P$ value of $\leq 0.05$ was considered statistically significant. Following univariable analyses, significant factors were subjected to a stepwise multivariable logistic regression analysis to determine variables associated with LOS using a $P$ value
Table 1

Demographic Features of the Study Group with Univariable Relationship of Each Preoperative Factor with Length of Stay.

\begin{tabular}{lllr}
\hline Feature & \multicolumn{1}{c}{ LOS $\leq 3$} & \multicolumn{1}{c}{ LOS $>3$} & P Value \\
\hline N & 221 & 39 & \\
Age & $63.9 \pm 9.1$ & $66.1 \pm 8.3$ & 0.164 \\
Gender & & & 0.326 \\
$\quad$ Male & $98(44.3 \%)$ & $4(35.9 \%)$ & \\
$\quad$ Female & $123(55.7 \%)$ & $25(64.1 \%)$ & \\
BMI & $32.2 \pm 6.1$ & $32.6 \pm 7.4$ & 0.704 \\
Pain level & $3.6 \pm 3.1$ & $4.0 \pm 3.1$ & 0.704 \\
Comorbidities & $2.9 \pm 1.9$ & $3.6 \pm 2.5$ & 0.032 \\
Use of assistive device & $70(31.7 \%)$ & $19(48.7 \%)$ & 0.039 \\
History of ECF admission & $2(0.9 \%)$ & $0(0.0 \%)$ & 1.000 \\
History of THA & $13(5.9 \%)$ & $3(7.7 \%)$ & 0.716 \\
History of TKA & $49(22.2 \%)$ & $8(20.5 \%)$ & 0.817 \\
Bilateral surgery & $3(1.4 \%)$ & $4(10.3 \%)$ & 0.011 \\
Assistance at home & & & $<0.001$ \\
$\quad$ Adequate & $171(77.4 \%)$ & $20(51.3 \%)$ & \\
$\quad$ Inadequate & $50(22.6 \%)$ & $19(48.7 \%)$ & \\
Expectation of discharge destination & & & $<0.001$ \\
Home & $163(74.1 \%)$ & $14(37.8 \%)$ & \\
ECF & $57(25.9 \%)$ & $23(62.2 \%)$ & \\
\hline
\end{tabular}

$\mathrm{ECF}=$ extended care facility.

$\leq 0.05$ to remain in the model. The linearity assumption between each continuous variable and the logit of the probability that LOS $>3$ was verified and transformations made if necessary. Concordance index (Cindex) was used to determine how well the model discriminated between different responses. SAS version 9.2 was used for all analyses. The conditional power of the study given the sample size and odds ratio of significant multivariable model covariates was reviewed for adequacy.

\section{Results}

Two hundred and sixty patients were included in this study. There were 112 males and 148 females with a mean age of 64.0 years (range 29-92). The mean BMI, pain level, and number of comorbidities were 32.3 (range $18.8-50.8$ ), 3.7 (range $0-10$ ), and 3.0 (range $0-9$ ) respectively. All patients were admitted from home and only $2(0.8 \%)$ patients had prior admission to an ECF. 89 patients (34.2\%) used assistive devices prior to surgery. 7 patients (2.7\%) underwent bilateral TKA. 69 patients (26.5\%) had a prior lower extremity total joint arthroplasty with TKA in the contralateral extremity accounting for $82.6 \%$ of those cases. 216 patients (83.1\%) had adequate caregiver assistance at home. The demographic features of the study cohort are outlined in Table 1.

The mean LOS was 3.0 days (range $2-7$ ). 173 patients (66.5\%) were discharged to home and 87 (33.5\%) were discharged to ECF, such as skilled nursing and acute rehabilitation facilities. Factors that were not significantly associated with LOS were age $(P=0.164)$, gender $(P=$ $0.326)$, BMI $(P=0.704)$, pain level $(P=0.434)$, prior TKA in the contralateral side $(P=0.817)$, and prior admission to an $\operatorname{ECF}(P=1.000)$. Number of comorbidities $(P=0.032)$, bilateral surgery $(P=0.011)$, use of assistive devices $(P=0.039)$, caregiver assistance at home $(P<0.001)$, and patient expectation of discharge destination $(P<0.001)$ were significant univariable preoperative factors. However, after multivariable adjustment, only number of comorbidities, caregiver assistance at home, and bilateral surgery remained significant in the reduced model. The Cindex for the multivariable logistic regression model was 0.815 . The multivariable model had more than $80 \%$ power to detect a significant increase

Table 2

Multivariable Logistic Regression Model.

\begin{tabular}{lrc}
\hline Factor & $P$ Value & Odds Ratio (95\% Confidence Interval) \\
\hline Comorbidities & 0.036 & $1.22(1.01-1.47)$ \\
Inadequate caregiver assistance & $<0.001$ & $8.36(3.77-18.54)$ \\
Bilateral surgery & 0.003 & $14.00(2.41-81.36)$ \\
\hline
\end{tabular}

$\mathrm{ECF}=$ extended care facility. 
Estimated Spline Transformation and 95\% C.I.

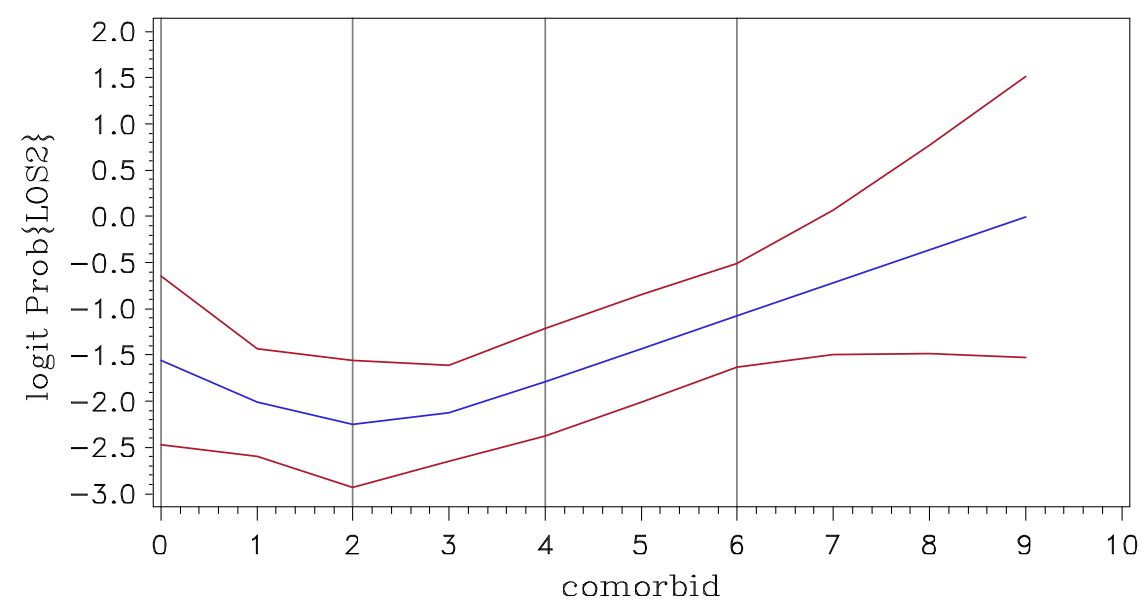

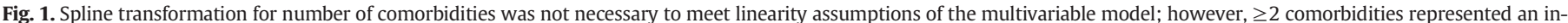
creased risk for longer length of stay.

in LOS adjusted for caregiver assistance at home $(P<0.001)$ and bilateral surgery $(P=0.003)$; however, comorbidities $(P=0.036)$ though significantly correlated with LOS were underpowered. Table 1 outlines the univariable relationship of each preoperative factor with LOS. Table 2 outlines the significant preoperative predictors in the final multivariable logistic regression model. Spline transformation for number of comorbidities was not necessary to meet linearity assumptions of the multivariable model; however, $\geq 2$ comorbidities represented an increased risk for longer LOS (Fig. 1).

Thirteen patients (5.0\%) developed minor postoperative complications during hospitalization, which were predominately narcoticrelated adverse drug events (e.g., nausea, hypotension, sedation, and confusion). There was a significant association between LOS and the development of a complication $(P<0.001)$. Eleven patients $(4.2 \%)$ had blood transfusion; however, there was no significant relationship between LOS and blood transfusion $(P=0.065)$. Patients with longer LOS had significantly higher rate of discharge to ECF $(P<0.001)$.

\section{Discussion}

Preoperative prediction of LOS following TKA is important for operational efficiency, management of patient expectations, and reduction of healthcare costs. Although several studies have been published, the relatively long LOS of those studies combined with broad exclusion criteria and variable postoperative rehabilitation protocols limits their application to current fast-track total knee arthroplasty practice. These differences in study design may explain some of the discrepancies between previous findings and ours. In particular, we did not find an association between LOS and age [11-14], gender [12], or use of assistive devices [11,12,14].

Husted et al retrospectively reviewed 342 TKA and found a relationship between LOS and age, gender, marital status, use of assistive devices, ASA score, and day of surgery [12]. While the multivariable logistic regression model in the Husted et al study was based on a control LOS $\leq 3$ similar to our study, the discharge criteria in that study were geared toward home and no physical therapy was provided on weekends. Weekend physical therapy has been shown to significantly decrease the LOS following TKA [16]. In addition, while the use of assistive devices may be important in postoperative rehabilitation protocols aimed toward discharge to home, it may not be equally important in today's fast-track arthroplasty practice where patients requiring longer postoperative care can be discharged to ECF. Therapists in ECF provide continued assistance in gait training, range of motion exercises, and other modalities that may lessen patients' dependence on assistive devices. The capacity for disposition to ECF may also explain the lack of association of LOS with age and gender in our study. Because these factors have been shown to increase the probability of discharge to ECF $[17,18]$, it may be understandable why we could not identify a relationship with age and gender as $33.5 \%$ of our patients were discharged to ECF; and hence were not captured in our analysis.

Styron et al performed the first study to examine the predictors of LOS after TKA in a U.S. patient population. This was a cross-sectional analysis of a national database that found surgeon volume and medical comorbidities to be significantly associated with LOS [15]. Medical comorbidities were measured by the Charlson comorbidity index. Patients undergoing concurrent total joint arthroplasty (TJA), those with previous TJA, and those in the outlier range of LOS were excluded. If we were to apply such exclusion criteria, nearly one-third of our patient population would have been eliminated. Interestingly, comorbidities remained a significant predictor of LOS in our study despite being assessed differently (number of active comorbidities vs. Charlson comorbidity index). The relationship between comorbidities and LOS has been established in other studies [12-14]. Given the intensive rehabilitation required after TKA, medical comorbidities can compound disability and limit functional recovery.

The comorbidities associated with LOS were quite variable and included both physical and mental conditions. While previous studies focused on select comorbidities [14], or used Charlson comorbidity index [15], we included all active comorbidities (physical and mental), which we believe is one of the strengths of the study. There is currently no gold-standard instrument for evaluating comorbidities; and even the most widely used scoring systems (American Society of Anesthesiologists score and Charlson comorbidity index) are not all-inclusive or have moderate inter-rater reliability $[19,20]$. For example, while the Charlson comorbidity index, initially designed as a predictor for mortality [21], is an excellent prognostic tool in life-threatening conditions [22], it may not be sensitive enough for low-mortality elective procedures [23] and it does not account for conditions that may impact the postoperative course, such as depression and anxiety. These conditions were common among patients with prolonged LOS in our study.

Our study has confirmed other previously reported risk factors for longer LOS, particularly inadequate caregiver assistance at home and bilateral surgery [14]. The demanding rehabilitation needs after TKA may explain the relationship between LOS and bilateral TKA, which imparts higher disability. In addition, inadequate caregiver assistance at home may confer decreased patient self-confidence and sense of security. Caregivers provide motivation to patients, encourage or aid in their ambulation while in the hospital, and instill greater reassurance among the ancillary staff about the safety of discharge.

We recognize that the retrospective nature of this study may represent a weakness. However, compared to a prospective study, a retrospective design may offer the advantage of eliminating observer bias as the 
data were collected without the intent of the study at that time. An outcome such as LOS could have been easily influenced by the study personnel if prospectively assessed. While our patient sample is small compared to previously published studies that were based on large-scale database searches, we had sufficient statistical power for all factors in the reduced multivariable logistic regression model, except for medical comorbidities. Given the very small contribution of comorbidities to increased LOS compared to caregiver assistance and bilateral surgery $(\mathrm{OR}=1.22 \mathrm{vs}$. 8.36 and 13.99 respectively), we did not feel that expanding the number of patients to power for this variable was necessary. In addition, the C-index, which is a measure of how well the multivariable logistic regression model discriminated between different responses, was quite good at 0.815 . We analyzed a consecutive sample of patients who underwent primary TKA without exclusion criteria. The target discharge date on the third postoperative day reflects current national trends. Patients received a standardized postoperative care protocol with clear discharge criteria. All the preoperative factors are easily obtainable with no need for scoring systems. Lastly, by reviewing individual patient charts, we had the advantage of assessing factors that were often not routinely collected in large-scale databases, such as caregiver assistance, patient expectations, and pain level. While we did not find a statistically significant multivariable contribution of preoperative pain level and patient expectations to LOS, this was the first study to examine the association of these factors with LOS.

This study has demonstrated that prolonged LOS following TKA is associated with increased number of comorbidities, lack of adequate caregiver assistance at home, and bilateral surgery. These factors may help identify high-risk patients so they may be appropriately counseled preoperatively, and so hospital staff may anticipate the need for escalated care, potentially reducing LOS. Furthermore, since patients with long LOS had significantly higher rate of discharge to ECF, early prediction of those who will require longer hospitalization may be used by case managers for proactive discharge planning. The positive correlation between discharge to ECF and the risk factors identified in this study (patient comorbidity, lack of caregiver assistance, and bilateral TKA) was previously demonstrated [17].

Finally, our study has unintentionally identified two targets for intervention to reduce LOS, particularly the prevention of narcoticsrelated adverse events and improvement of preoperative patient education. Our group has recently implemented a new pain management pathway emphasizing preemptive and multimodal analgesia to decrease the reliance on narcotics. We have also implemented an enhanced preoperative patient education program where discharge destination is now determined at time of preoperative clinic visit and not postoperatively. This education program incorporates all members of the care team including nurses, therapists, physician-assistants, and physicians. By adopting these two measures, we have noticed a dramatic decline in LOS following TKA similar to previous reports [8,24].

\section{References}

1. Daigle ME, Weinstein AM, Katz JN, et al. The cost-effectiveness of total joint arthroplasty: a systematic review of published literature. Best Pract Res Clin Rheumatol 2012;26(5):649.

2. Kurtz S, Ong K, Lau E, et al. Projections of primary and revision hip and knee arthroplasty in the United States from 2005 to 2030. J Bone Joint Surg Am 2007;89 (4):780.

3. National Center for Health Statistics. Hospitals b, and occupancy rates, by type of ownership and size of hospital: United States, selected years 1975-2009; 2011 [Hyattsville, Maryland]

4. Bazzoli GJ, Brewster LR, Liu G, et al. Does U.S. hospital capacity need to be expanded? Health Aff 2003:22(6):40.

5. King J, Stamper DL, Schaad DC, et al. Minimally invasive total knee arthroplasty compared with traditional total knee arthroplasty. Assessment of the learning curve and the postoperative recuperative period. J Bone Joint Surg Am 2007:89(7):1497.

6. Malviya A, Martin K, Harper I, et al. Enhanced recovery program for hip and knee replacement reduces death rate. Acta Orthop 2011;82(5):577.

7. Tayrose G, Newman D, Slover J, et al. Rapid mobilization decreases length-of-stay in joint replacement patients. Bull Hosp Joint Dis 2013;71(3):222.

8. Duellman TJ, Gaffigan C, Milbrandt JC, et al. Multi-modal, pre-emptive analgesia decreases the length of hospital stay following total joint arthroplasty. Orthopedics 2009;32(3):167.

9. Kahn KL, Rubenstein LV, Draper D, et al. The effects of the DRG-based prospective payment system on quality of care for hospitalized Medicare patients. An introduction to the series. JAMA 1990;264(15):1953.

10. Cram P, Lu X, Kates SL, et al. Total knee arthroplasty volume, utilization, and outcomes among Medicare beneficiaries, 1991-2010. JAMA 2012;308(12):1227.

11. Smith ID, Elton R, Ballantyne JA, et al. Pre-operative predictors of the length of hospital stay in total knee replacement. J Bone Joint Surg 2008;90(11):1435.

12. Husted H, Holm G, Jacobsen S. Predictors of length of stay and patient satisfaction after hip and knee replacement surgery: fast-track experience in 712 patients. Acta Orthop 2008;79(2):168.

13. Jonas SC, Smith HK, Blair PS, et al. Factors influencing length of stay following primary total knee replacement in a UK specialist orthopaedic centre. Knee 2013;20(5):310

14. Ong PH, Pua YH. A prediction model for length of stay after total and unicompartmental knee replacement. Bone Joint J 2013:95-B(11):1490.

15. Styron JF, Koroukian SM, Klika AK, et al. Patient vs provider characteristics impacting hospital lengths of stay after total knee or hip arthroplasty. J Arthroplasty 2011;26 (8):1418 [e1411-1412].

16. Hughes K, Kuffner L, Dean B. Effect of weekend physical therapy treatment on postoperative length of stay following total hip and total knee arthroplasty. Physiother Can 1993;45(4):245.

17. Barsoum WK, Murray TG, Klika AK, et al. Predicting patient discharge disposition after total joint arthroplasty in the United States. J Arthroplasty 2010;25(6):885.

18. Bozic KJ, Wagie A, Naessens JM, et al. Predictors of discharge to an inpatient extended care facility after total hip or knee arthroplasty. J Arthroplasty 2006;21(6 Suppl 2):151.

19. Bjorgul K, Novicoff WM, Saleh KJ. Evaluating comorbidities in total hip and knee arthroplasty: available instruments. J Orthop Traumatol 2010;11(4):203.

20. Sankar A, Johnson SR, Beattie WS, et al. Reliability of the American Society of Anesthesiologists physical status scale in clinical practice. Br J Anaesth 2014;113(3):424.

21. Charlson ME, Pompei P, Ales KL, et al. A new method of classifying prognostic comorbidity in longitudinal studies: development and validation. J Chronic Dis 1987;40(5):373.

22. Kastner C, Armitage J, Kimble A, et al. The Charlson comorbidity score: a superior comorbidity assessment tool for the prostate cancer multidisciplinary meeting. Prostate Cancer Prostatic Dis 2006;9(3):270.

23. Harse JD, Holman CD. Charlson's Index was a poor predictor of quality of life outcomes in a study of patients following joint replacement surgery. J Clin Epidemiol 2005;58(11):1142.

24. Yoon RS, Nellans KW, Geller JA, et al. Patient education before hip or knee arthroplasty lowers length of stay. J Arthroplasty 2010;25(4):547. 